\title{
From cognition to biomechanics and back: The end-state comfort effect and the middle-is-faster effect ${ }^{1}$
}

\author{
David A. Rosenbaum ${ }^{\mathrm{a}, *}$, Caroline M. van Heugten ${ }^{\mathrm{b}}$, \\ Graham E. Caldwell ${ }^{\circ}$ \\ a Department of Psychology, Moore Building, Pennsylvania State University, University Park, PA 16802, USA \\ ${ }^{\mathrm{b}}$ Department of Psychology, University of Leiden, Leiden, The Netherlands \\ ${ }^{c}$ Department of Exercise Science, University of Massachusetts, Amherst, MA, USA
}

Received 11 January 1995; revised 15 September 1995; accepted 9 November 1995

\begin{abstract}
Consistent preferences for particular types of movement suggest criteria for movement selection. These can be important when, as is usually the case, infinitely many movements allow a task to be achieved. The experiments reported here were designed to identify the source of a strong preference observed in earlier object-manipulation studies. In those earlier studies, subjects usually grabbed objects to be moved from one location to another in a way that afforded a comfortable final posture rather than a comfortable initial posture (the end-state comfort effect). The comfortable final state usually allowed the forearm to be at or near the middle of its range of motion on the pronation-supination dimension. The hypothesis tested here was that the end-state comfort effect stemmed from an expectation that movements can be made more quickly in the middle of the pronation-supination range than at either extreme. To test this hypothesis, we asked subjects, in the first experiment, to perform a handle rotation task that demanded little or no precision and so no need to make rapid to-and-fro homing-in movements near the end of the rotation. Half the subjects did not show the end-state comfort effect, in contrast to all previous studies, where all subjects showed the effect. An incidental finding of the first experiment was that handle rotations that ended at or near the end of the range of motion took longer than handle rotations that ended at or near the middle of the range of motion. To test the latter result more

\footnotetext{
*Corresponding author. E-mail: dar12@cac.psu.edu, Fax: + 1814 863-7002, Tel.: + 1814 863-1991.

1 These experiments were conducted while the second author completed an internship with the first author at the University of Massachusetts, Amherst. The studies were described in a thesis submitted by the second author to the University of Leiden in partial satisfaction of the Doctorandus degree.
} 
carefully, we asked subjects, in Experiments 2 and 3, to oscillate the forearm as quickly as possible, either in the supination part of the forearm rotation range, in the middle part of the range, or in the pronation part of the range. As predicted, oscillation frequencies were highest in midrange, and this was true for both hands. The results as a whole have implications for the relation between cognitive psychology and biomechanics, and for human factors.

Keywords: Motor processes; Movement speed; Handedness

\section{Introduction}

As is obvious from patients with movement disorders, neophyte performers, and most modern robots, motor control is far from a trivial problem. One can appreciate its difficulty by considering a basic fact of motor performance: Virtually every task has an infinite number of motor solutions. Yet single solutions are normally found in little time and with little effort. The research described here bears on the question of how such solutions are found.

It is useful to consider the kinds of answers that have been given to the question of how movements are selected. One answer, originating with von Holst (1939) and Bernstein (1967), is that dependencies between or among components of the musculoskeletal system help reduce the effective number of degrees of freedom to be managed (Kugler et al., 1980). Thus, fewer movement possibilities exist than might be supposed if one simply counted ostensibly independent elements. Such dependencies, or synergies, can be found by identifying interactions between or among simultaneously performed activities (Kugler et al., 1980).

Another answer to the question of how movements are selected is that biomechanical properties of the musculo-skeletal system obviate detailed motor planning. For example, spring-like properties of muscles can allow limb motions to be generated without detailed computations of the trajectories to be followed (Bizzi and Mussa-Ivaldi, 1989; Thelen et al., 1987). Similarly, gravitational forces can ensure completion of some aspects of movement with little or no executive control (McMahon, 1984).

A third answer to the question of how movements are selected is motivated by the belief that neither of the above answers is sufficient. That is, although synergies and mechanics may simplify the problem of movement selection, neither factor can always solve it. For example, the fact that one can pick up a book with the left or right hand, but chooses to use one hand, indicates that choices are not fully limited by synergies or mechanical constraints. The fact that one is more likely to pick up a book with one's left hand if the book is farther to the left indicates that there are selection criteria which play a role in movement choices. These criteria may be 'hard' in the sense that they require particular choices, or 'soft' in the sense that they allow for different choices depending on the performance context. Allowing for selection criteria raises the question of what the criteria are and where they originate. The experiments reported here bear on this question within the context of a behavioral phenomenon uncovered earlier by the first author and his colleagues. The phenomenon is reviewed below, followed by new experiments designed to identify its source. In the last part of the article, we consider the 
implications of our research for the relation between cognition and biomechanics. Historically, these two fields have been largely divorced but, as seen here, they deserve and indeed need to be considered together.

\section{The end-state comfort effect}

\subsection{The experimental procedures}

Consider the simple task of reaching for a bar (see Fig. 1). Suppose the instructions to the subject indicate that the bar should be picked up with the right hand and should be grabbed firmly like a tennis racquet, and that the right end of the bar (the white end in Fig. 1) should be placed on a target to the left of the stand on which it is resting. When twelve subjects performed this task (Rosenbaum et al., 1990), all of them picked up the bar with an overhand grip - that is, with the thumb pointing to the left side of the bar. Likewise, all twelve subjects picked up the bar with an overhand grip when the same end (the right end) was to be placed on a target to the right of the stand. However, when the same twelve subjects were asked to place the opposite end of the bar (the left end) either to the left or to the right target, all of them used an underhand grip, picking up the bar with the thumb pointing to the right side. In all conditions subjects could have grabbed the bar with either grip. The fact that subjects made different choices in

(A)

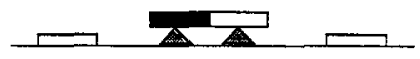

(B)

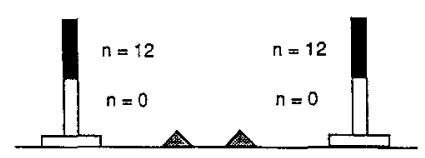

(C)

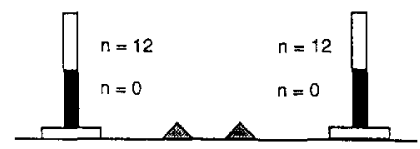

Fig. 1. The bar transport study of Rosenbaum et al. (1990). (A) The experimental setup, consisting of a bar resting on a stand. (B) Number of subjects, $n$, who grabbed the bar with the thumb toward the black end or white end when the white end was brought to the target on the left or right. (C) Number of subjects, $n$, who grabbed the bar with the thumb toward the black end or white end when the black end was brought to the target on the left or right. Adapted from D.A. Rosenbaum, J. Vaughan, H.J. Barnes, F. Marchak and J. Slotta, 1990. 'Constraints on action selection: Overhand versus underhand grips'. In: M. Jeannerod (Ed.), Attention and performance XIII (pp. 321-342). Hillsdale, NJ: Erlbaum. 
different tasks indicates that they relied on one or more selection criteria to determine how the task should be performed.

In analyzing subjects' performance, it was possible to determine what the selection criterion was, at least in general terms: Subjects sought to perform the task in a way that ensured a comfortable final posture. This conclusion was supported by psychophysical ratings. When independent raters evaluated the comfort of the overhand and underhand grips, both at the initial (horizontal) and final (vertical) bar locations, they consistently gave higher comfort ratings to the overhand grips (thumb toward the black end in Fig. 1) than to the underhand grips (thumb toward the white end in Fig. 1). It turned out that the best predictor of grip choices were ratings of final posture comfort (how comfortable the grip would be when the bar was in the vertical position), rather than ratings of initial posture comfort (how comfortable the grip would be when the bar was in the horizontal position), or mean ratings of initial- and final-posture comfort. Thus, subjects emphasized comfort in the terminal phase of the task. This conclusion was further supported by patterns of grip choices when the task was reversed. When the bar stood vertically on one of the flat targets and had to be placed on the horizontal stand so the bottom end of the bar would lie on the stand's left end, subjects grabbed the bar with the posture they avoided before: They grabbed the bar with the thumb pointing down, which was the posture they avoided when placing the bar vertically on the target after removing it from the horizontal stand. Grabbing the erect bar with the thumb pointing down made it possible to complete the task in a comfortable posture (the overhand grip). Rosenbaum et al. (1990) called this preference for ending comfortably the end-state comfort effect.

To further investigate the end-state comfort effect, Rosenbaum et al. (1993) developed a task that allowed for continuous measures of grip preferences. They mounted a handle on a freely rotating wheel and spaced eight targets around the edge of the wheel like the numbers on a clock face (see Fig. 2A). Subjects were asked to grab the handle and turn it, causing a tab on one end to be aligned with a target designated by the experimenter at the start of the trial. The main dependent measure was the grip subjects spontaneously adopted: Would they grab the handle with the thumb on the same side of the handle as the tab or on the opposite side? The result, reproduced in Fig. 2B, was that the chosen grip depended on where the tab ultimately had to go. Again, subjects selected the grip (thumb toward or away from the tab) that afforded a comfortable final posture. This effect held up when the left hand was tested as well as when the right was tested (Rosenbaum et al., 1992) and it held up when performance was evaluated under reaction-time pressure (Rosenbaum et al., 1992).

\subsection{Possible explanations of the end-state comfort effect}

Why did subjects exhibit the end-state comfort effect? Clearly, they planned beyond the first grip, anticipating future states. But what factors did they take into account in the planning process? A number of hypotheses were considered, as seen below.

\subsubsection{Exploitation of gravity}

One possibility was that the end-state comfort effect derived from exploitation of gravity. Observation of the postures that subjects adopted during the handle rotation task indicated that in twisting the arm to the more uncomfortable starting posture, they raised 


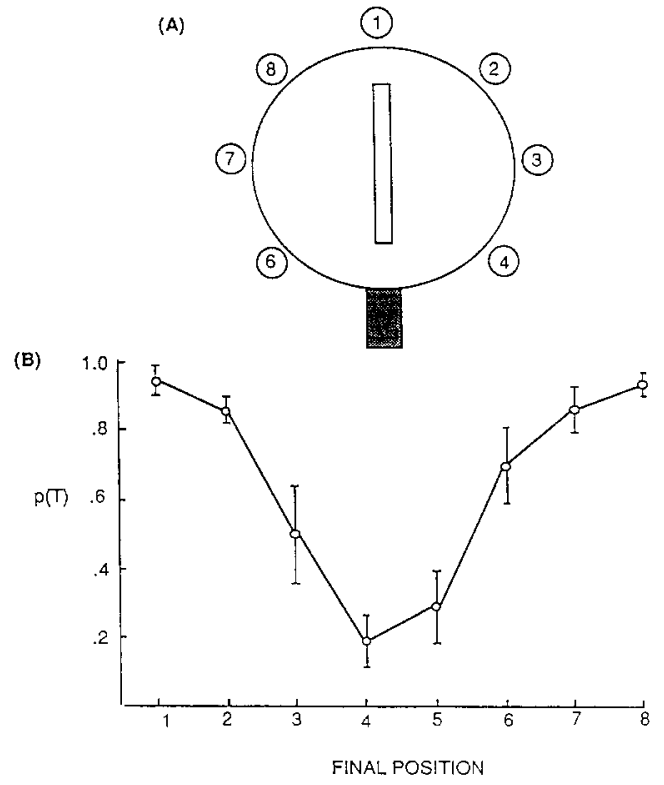

Fig. 2. The handle rotation study of Rosenbaum et al. (1992). (A) The main apparatus as seen by the subject. The rectangle corresponds to a handle protruding from the wheel. Turning the handle caused the wheel to turn. A tab extending from the plane of the wheel turned with the wheel, and could cover any of the numbers appearing slightly below the wheel's plane of rotation; in this case, the tab covers the number 5. (B) Probability, $p(T)$, of grabbing the handle with the thumb toward the tab as a function of the tab's final position. Standard error bars reflect variability associated with starting position. The task was performed with the right hand. From D.A. Rosenbaum, J. Vaughan, M.J. Jorgensen, H.J. Barnes and E. Stewart, 1993. 'Plans for object manipulation'. In: D.E. Meyer and S. Kornblum (Eds.). Attention and Performance XIV - A silver jubilee: Synergies in experimental psychology, artificial intelligence and cognitive neuroscience (pp. 803-820). Cambridge, MA: MIT Press/Bradford Books.

the elbow, and then, during the handle rotation movement, allowed the elbow to drop. Conceivably, subjects set themselves in an uncomfortable initial posture so the handleturning movement would be aided by gravity.

To test this hypothesis, Rosenbaum et al. (1993) placed the handle-rotation device on the floor and allowed subjects to perform the task with their arms hanging down. The idea was that if the end-state comfort effect depended on dropping the shoulder and elbow, the effect would disappear in the vertical orientation. The results were virtually identical to what they were before, however, contrary to the gravity hypothesis. The failure to support the gravity hypothesis fits with the fact in some bar-transport tasks subjects exhibited the end-state comfort effect even when bringing the bar to a target position above the head (Rosenbaum and Jorgensen, 1992). The latter result is hard to reconcile with a gravity-based explanation.

\subsubsection{Use of elastic energy}

Another possibility is that people 'wound up the arm' like a spring, taking hold of the handle in an awkward posture and then releasing the arm, allowing it to unwind 
passively, or be aided by such passive unwinding, on its way to a rest position. According to this elastic-energy hypothesis, subjects exploited elastic energy, stored during the initial phase when grabbing the bar or handle, and permitted the arm to recoil during the rotation or transport task. This strategy would have permitted exploitation of the mechanics of the musculo-skeletal system, thereby reducing the computational burden on the motor planning system (Bizzi and Mussa-Ivaldi, 1989; Thelen et al., 1987). Such a scenario is plausible in view of the fact that muscle elasticity has been implicated in enhancing efficiency in tasks such as jumping and running (Alexander, 1984; McMahon, 1984). In much the same way that it helps vertical jumping first to drop down and then 'bounce up', it may have helped subjects to rotate the arm in the opposite direction to the one they ultimately used for the main rotation task, in which case ending comfortably was just a correlate of the main performance goal. (For a review of the role of elasticity in muscle function, see Shorten, 1987.)

Despite the plausibility of the elastic energy hypothesis, we believe it has only limited importance. The reason is that the end-state comfort effect appeared even in complex, multi-joint movements for which it was doubtful that elastic energy was useful. For example, when subjects carried a bar to a target above their heads or brought a bar down by their knees, they showed the end-state comfort effect just as strongly as in the tasks requiring simple pronation/supination movements of the horizontally extended forearm (Rosenbaum and Jorgensen, 1992). Because it is doubtful that elastic energy would have been as useful for these larger-scale vertical movements as they were for the smaller-scale horizontal movements, it is unlikely that exploitation of stored elastic energy was the main cause of the end-state comfort effect.

\subsubsection{Precision}

The most promising explanation of the end state comfort effect is that it is easier to make positioning movements at or near the middle of the range of motion than at or near the extremes; we call this the precision hypothesis. ${ }^{2}$ The capacity for moving quickly is helpful in positioning tasks, at least when actors place a premium on both speed and accuracy. In terms of Fitts' Law (Fitts, 1954), which states that movement time is a linear function of the logarithm of the ratio of the distance to be covered relative to the spatial tolerance around the target point, movement time will be minimized when the slope of this linear function approaches zero. According to the precision hypothesis, this slope may be nonuniform over the range of angles that the joints can occupy; in particular, the slope may be lower in the middle of the range of motion than at the extremes. If subjects in the original bar transport and handle rotation studies capitalized on this feature of performance, they would have chosen grips that afforded midrange

\footnotetext{
${ }^{2}$ The range being referred to is pronation-supination of the forearm. Pronation-supination is defined with respect to rotation of the forearm about its long axis. Looking down at one's own right forearm when it is held in a horizontal position and in the sagittal plane, one sees the forearm fully pronated when the thumb is turned as far as possible in the counterclockwise direction (palm facing down), and one sees the forearm fully supinated when the thumb is turned as far as possible in the clockwise direction (palm facing up).
} 
final postures rather than extreme final postures in order to complete the most time-consuming part of aiming performing (homing in) in the least amount of time. ${ }^{3}$

This explanation is compatible with all the observations made about the end-state comfort effect so far. In the bar transport tasks, subjects had to place the bar on a target which was only slightly wider than the bar's diameter. Similarly, in the handle rotation tasks, subjects had to line up the pointer with a target that had relatively small tolerance for error. Moreover, in the handle rotation tasks the wheel on which the handle was mounted had very low inertia, friction, and damping. Thus, subjects had to exert very careful control over the wheel's final position.

These observations do not constitute direct tests of the precision hypothesis, however; they are only retrospective accounts of task features that are compatible with the hypothesis. The present study was designed to provide a stronger test of the precision hypothesis. The first experiment tested a crucial prediction of the hypothesis - namely, that the end-state comfort effect should be small or even absent when precision requirements are low or nonexistent. As will be seen here, this prediction was confirmed. The second and third experiments provided further tests of the precision hypothesis and confirmed that movements can indeed be made more quickly within the middle of the range of motion than at or near the extremes. As far as we know, this is a newly discovered phenomenon of motor performance. We call it the middle-is-faster effect. The fact that we were led to postulate this effect based on macroscopic features of people's behavior (how they chose to grab an object to be moved) highlights the importance of links between cognition and biomechanics. We comment on those links in the final section of the article, where we also consider the implications of this research for human factors.

\section{Experiment 1}

As stated above, a critical prediction of the precision hypothesis is that the end-state comfort effect should disappear when precision requirements are removed. Said differently, if the end-state comfort effect reflects subjects' desire to promote precise positioning at the end of forthcoming manipulations, then when the final positioning does not demand precision, the end-state comfort effect should not emerge.

\footnotetext{
${ }^{3}$ That the homing-in phase of aiming is the most time-consuming part of aiming is demonstrated by the fact that for a long time it was believed that the Crossman and Goodeve (1963) account of Fitts' Law was correct. According to Crossman and Goodeve's model, aiming for a target is achieved by making a series of movements, each of which covers a constant proportion of the distance to the center of the target and each of which occurs in a constant amount of time. Thus, the first half of the remaining distance might be covered in the first time step, then half of the still-remaining distance would be covered in the next time step (i.e., one quarter of the total distance that had to be covered in the beginning), and so forth. Although Crossman and Goodeve's account has been replaced by other, more powerful theories, most notably the model of Meyer et al. (1988), Crossman and Goodeve's account as well as its successors captures the basic feature of aiming performance that the final homing-in phase of aiming takes disproportinately more time than the initial 'ballistic' phase.
} 


\subsection{Method}

The low-precision prediction was tested by modifying the handle rotation device used in the previous studies. Whereas in the previous handle rotation studies, subjects always had to bring the handle to a final specified orientation, with the modified device used here, subjects merely had to rotate the handle past a designated target. The way subjects could tell whether they had turned the handle a sufficient distance was that a bolt, mounted to the rim of the wheel turned by the handle, dropped passively into a hole at the target location when the handle turned past that location. To allow the bolt to drop into the hole, the modified handle-rotation device was placed on the floor, as in the 'gravity study' of Rosenbaum et al. (1993). As in the gravity study, subjects were seated and could grab the horizontally oriented handle either with the thumb pointing toward or away from a pointer on one end of the handle. Mechanically, the handle and wheel were essentially the same as the handle and wheel used in the study of Rosenbaum et al. (1993). The only difference was that the bolt was mounted on the outer rim of the wheel turned by the handle. When the wheel was turned, the bolt slid along a low-friction circular track, and when the bolt passed over a hole in the track, it dropped into the hole. The track itself could be turned by the experimenter to vary the position of the hole, which was conspicuously marked with a red numbered tab, $2.54 \mathrm{~cm}$ wide. The red tab served both to locate the hole for the subject and to allow the experimenter to align the hole with one of eight fixed positions on the base of the apparatus. These were spaced evenly around the outer edge of the circular track, as seen in Fig. 3.

The subject began each trial sitting with his or her legs apart looking down at the device, and with his or her forearms resting on the thighs. When the experimenter confirmed that the subject was sitting correctly, he turned the circular track to bring it to the next test position and then turned the handle so the pointer was $180^{\circ}$ away from the target position (the position of the hole). The experimenter took care during these

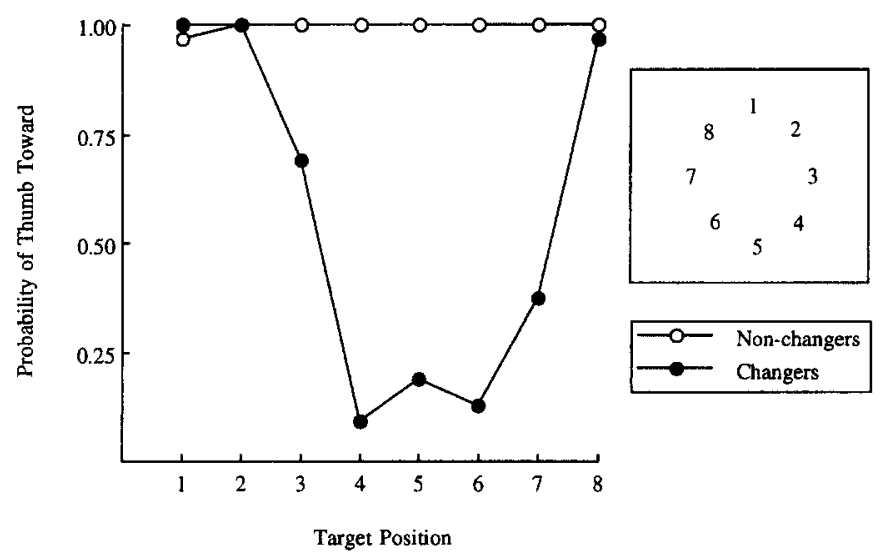

Fig. 3. Probabilty of of grabbing the handle with the thumb toward the pointer, as a function of the end position to which the pointer had to be brought, for the subjects who spontaneously switched grips and for the subjects who did not. Inset identifies position numbers. Data from Experiment 1. 
manipulations to manipulate the circular track and handle with two hands rather than one, so subjects would not be biased in the way they grabbed the handle themselves.

At the beginning of the experimental session, subjects were shown how the apparatus worked, although again the experimenter took care to manipulate the apparatus with two hands rather than one. Subjects were told that they had to take hold of the handle firmly like a tennis racquet and hold onto the handle throughout the rotation; these instructions were the same as in Rosenbaum et al. (1993). In addition, subjects were told that all they had to do was 'give the wheel a gentle whirl' to get the wheel turning to enable the bolt to drop into the hole. Subjects were told that the task would be easiest if the wheel was turned at moderate speed and that the purpose of the experiment was just to see what people do when performing this task in a relaxed, natural fashion.

Each of the eight positions was tested eight times for each subject, with the order of positions balanced according to a Latin square design. The experiment was videotaped. Eight undergraduate students, four male and four female, from the University of Massachusetts served as subjects in exchange for class credit. The students ranged in age from 19 to 27 years. All were right-handed.

\subsection{Results and discussion}

\subsubsection{Grip choices}

As in the earlier handle rotation studies, the primary data of interest concerned subjects' grip choices: Would subjects grab the handle with the thumb toward the pointer ('thumb-toward') or with the thumb away from the pointer ('thumb-away'), and how would the likelihood of adopting one grip or the other depend on the target position to which the pointer had to be brought? (We assumed that the final position was the critical variable, rather than the starting position, based on the earlier studies.) Subjects' grip choices were noted from the videotape and the number of thumb-toward grips for each target orientation were tallied for each subject. Direction of rotation was also noted.

The grip-choice results were strikingly different from the results of any previous grip-choice study. As seen in Fig. 3, a dramatic individual difference emerged: Only half the subjects changed their grips depending on the task. The other subjects used the same hand grip in all (or virtually all) conditions. The way these results differ from the previous experiments is that in all previous studies, all subjects altered their grip depending on the manipulation to be performed, and they did so by at least a factor of 2 ; that is, in all previous studies, every subject was at least twice as likely to use a thumb-toward grip for one task as for another.

In the present study, for those subjects who always used the same grip, the grip that was chosen was the one that brought the thumb to the same side of the handle as the pointer. These subjects exhibited a thumb-toward bias. This bias was seen in all the previous handle rotation studies reported by Rosenbaum and colleagues, but it was always tempered before by the end-state comfort effect. That is, the subjects in the previous experiments, like the subjects in the present experiment who switched grips, had a basic bias to grab the handle with the thumb toward the pointer, which was reflected in the fact that the minimum probability of grabbing the handle with the thumb toward the pointer was above zero. However, these subjects counteracted this bias to an 
increasing extent the more awkward the final hand positions would be. The subjects in the present experiment who switched grips, like the subjects in the earlier experiments, were least likely to grab the handle with the thumb toward the pointer when the forthcoming handle rotations would end near position 4 (see Fig. 3). This is the position where the forearm is closest to the end of its pronation-supination range. The nonswitching subjects (those who always grabbed the handle with the thumb toward the pointer) were therefore willing to end in uncomfortable final postures, such as thumb toward position 4 . The fact that the end-state comfort effect vanished for some subjects provides support for the precision hypothesis, which predicted that there would be no end-state comfort when subjects had to grab the handle and rotate it with limited precision requirements.

Why did half the subjects still show the end-state comfort effect? Unfortunately, nothing in the subjects' personal information helps us discriminate the switchers from the nonswitchers. Half the switchers were male and half were female. Although 3 of the 4 nonswitchers were the first 3 subjects tested, the fourth switcher was the sixth subject tested, so there was no obvious artifact of the experimenter's experience with the task that accounted for the individual difference. Finally, the directions of the handle rotation were not different for the two groups, except insofar as subjects who grabbed the handle with the thumb toward or away from the pointer rotated the handle in the way that was anatomically possible. (Previous studies have shown that the end-state comfort effect is not attributable to preferred directions of rotation. For the end-state comfort effect to be possible, in fact, it must be the case that people are as likely to rotate the handle clockwise as counterclockwise.)

It is possible that the subjects who showed the end-state comfort effect saw the need to exercise more precise control over the handle in this task than was really needed. They may have believed that rotating the handle too quickly could have caused the bolt to ride past the hole (which was in fact not the case because the apparatus was designed so the bolt would drop into the hole for the rotation speeds that human subjects could produce). If these subjects thought that some slowing of the handle was necessary when the bolt approached the hole, it would have been reasonable for them to show the end-state comfort effect because they treated the task as one demanding precision.

\subsubsection{Handle rotation times}

The fact that some subjects switched grips in this experiment and others did not created an opportunity for us to evaluate the precision hypothesis in a new, initially unplanned, way. Because there were an appreciable number of handle rotations that brought the hand to uncomfortable final postures (postures at or near extreme positions) as well as handle rotations that brought the hand to comfortable final postures (postures far from extreme positions), it was possible to test the hypothesis that it took longer for the former handle rotations than for the latter handle rotations. That is, according to the precision hypothesis, one would expect rotations that ended in uncomfortable final postures to take longer than rotations that ended in comfortable final postures. It was not possible to test this hypothesis in earlier studies because there were very few handle rotations that ended in uncomfortable postures.

To test the prediction that rotations that ended in uncomfortable final postures would 
take longer than rotations that ended in comfortable final postures, the videotape of subjects' performance was subjected to a frame-by-frame analysis. For each trial, we identified the first frame in which the handle was turned from the starting position and the frame in which the bolt dropped into the hole. The difference in the number of frames provided an estimate of the duration of the rotation. Given that the video frame rate was only 16 frames per second, the resolution was only $0.0625 \mathrm{~s}$ per frame.

The most important test concerned those rotations that ended with the thumb toward position 4 versus those rotations that ended with the thumb toward position 8 (the position opposite position 4). Position 4 was the most uncomfortable final position, judging from the psychophysical function of the switching subjects (see Fig. 3). According to the precision hypothesis, rotations that ended with the thumb toward position 4 would take longer than rotations that ended with the thumb toward position 8 . There were 36 grips that ended with the thumb at position 4 compared to 90 grips that ended with the thumb at position 8 . The mean handle rotation time for the rotations that ended at position 4 was $0.99 \mathrm{~s}$, whereas the mean handle rotation time for the rotations that ended at position 8 was $0.86 \mathrm{~s}$. As tested with a $t$-test (one-tailed), this difference was reliable, $p<0.01(d f=124)$.

Regarding the handle rotations for which the thumb ended up at other positions, the lower video frame rate precluded any definitive conclusions, because all these mean handle rotations occupied a range of less than $0.0625 \mathrm{~s}$. In fact, only the handle rotations for which the thumb ended at position 4 were special. The mean of these rotation times was $0.0625 \mathrm{~s}$ longer than any other mean, though all the other means occupied a range of less than $0.0625 \mathrm{~s}$. Given the low temporal resolution of the video system, it would have been inappropriate to test differences among the times for the rotations that ended at positions other than 4 .

\section{Experiment 2}

The results of Experiment 1 provided some support for the precision hypothesis. Consistent with the hypothesis, when precision requirements were reduced via the bolt-drop device, the strength of the end-state comfort effect diminished. Only half the subjects showed the end-state comfort effect, whereas in previous studies, when the precision requirements were high, all subjects showed the effect. Also consistent with the precision hypothesis, handle rotation times were longest when subjects ended the handle rotations with the thumb pointing to the most uncomfortable final thumb position.

Despite these positive features of Experiment 1, it is problematic that all subjects did not abandon the end-state comfort effect; this outcome can only be understood in speculative terms (e.g., subjects thought they needed more control than was really needed). Therefore, a second experiment was designed to provide a stronger test of the precision hypothesis. The tack taken in the design of the second experiment was similar to the tack taken in the design of Experiment 1 in that there were minimal precision demands. This time, however, the precision demands were made even smaller than before and subjects did not have to choose a hand grip. All they had to do was oscillate 
the end of a hand-held stick as quickly as possible between two stops a small distance apart. In one condition, the movements were made with the forearm in the middle part of its range of motion, in another condition the movements were made with the forearm in the pronated part of its range of motion, and in a third condition the movements were made with the forearm in the supinated part of its range of motion. The prediction was that the frequency of oscillation would be highest when the oscillations were made in the middle of the range.

\subsection{Method}

\subsubsection{Subjects}

Twelve students, eight male and four female, from the University of Massachusetts, Amherst, volunteered. Their mean age was 26.2 years (range 21 to 36 years). Three subjects were left-handed (two males and one female); the other nine (six males and three females) were right-handed. All subjects reported normal or corrected-to-normal vision, and none reported neurological or motor disorders. All subjects were naive with respect to the scientific aims of the study.

\subsubsection{Apparatus and procedure}

As shown in Fig. 4, the subject performed the task in a standing position, holding the stick in the preferred hand, with the upper arm oriented vertically by the side, and the forearm held in the horizontal plane with the elbow stationary. Two switches were housed in a U-shaped wooden device which could be oriented so its open end faced the right in the pronation condition, down in the midrange condition, or the left in the

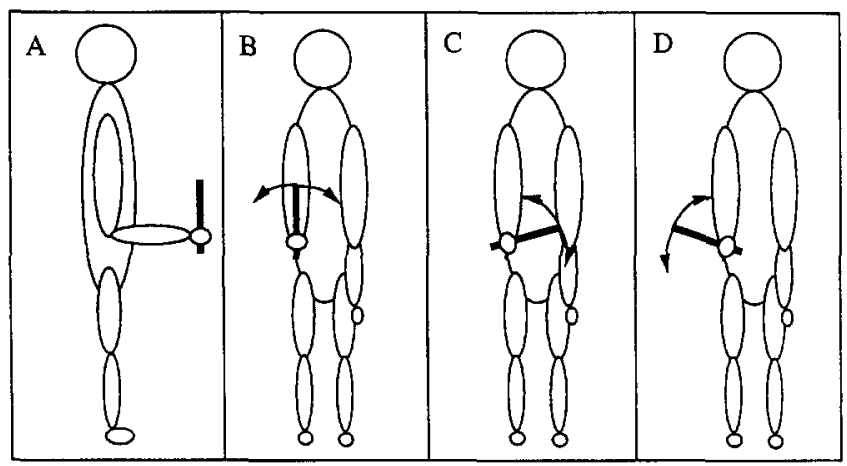

Fig. 4. Positions adopted by subjects in Experiments 2 and 3. (A) Side view. (B) Front view showing oscillation in the central position (middle of the pronation-supination range). (C) Front view showing oscillation in the medial position (pronation part of the pronation-supination range). (D) Front view showing oscillation in the lateral position (supination part of the pronation-supination range). The arrows in panels B-D show the sense of direction covered by the end of the hand-held stick, but the lengths of the arcs are much greater than the the lengths of the arcs actually covered by the end of the stick, simply for the sake of visual clarity. The microswitches struck by the end of the stick are not shown, nor are other parts of the apparatus. 
supination condition. The device was made of three rectangular pine blocks. Two pieces of wood $(2 \times 5 \times 10 \mathrm{~cm})$ formed the legs and the third formed the center $(2 \times 5 \times 10.35$ $\mathrm{cm}$ ). At the top interior of each leg was mounted a Unimax microswitch (part number 2 HBT/1). The switches were positioned so the distance between them was $10.35 \mathrm{~cm}$. The apparatus was clamped onto the metal shelf of a bookcase. The height of the shelf could be adjusted for each subject.

The stick was a maple dowel, $30 \mathrm{~cm}$ long, $1.2 \mathrm{~cm}$ in diameter, weighing $113 \mathrm{~g}$. One end of the dowel was held by the subject. The other end was tapped back and forth between the microswitches. The length of the dowel, the distance between the switches, and the orientations of the box were chosen so that movements between the switches corresponded to rotations between $15^{\circ}$ and $45^{\circ}$ (pronated part of the range), between $75^{\circ}$ and $105^{\circ}$ (midrange part of the range), and between $135^{\circ}$ and $165^{\circ}$ (supinated part of the range), where $0^{\circ}$ was defined as the fully pronated position and degrees increased with the amount of supination.

The microswitches were connected to a Strawberry Tree digital input board (Strawberry Tree, Inc., Sunnyvale, CA), interfaced to an Apple Macintosh II computer. The experiment was run with Strawberry Tree's Workbench software, as amplified with software developed in our lab (Bushe et al., 1994).

\subsubsection{Procedure}

Subjects were tested individually in sessions lasting about $15 \mathrm{~min}$. At the start of the experiment, subjects were given information about the task goals. The height of the apparatus was adjusted to ensure that the forearm was held horizontally, and the elbow was always in a $90^{\circ}$ angle. The subject stood in a relaxed upright position, facing the apparatus, with the frontal plane of the body parallel to the bookcase. The dowel was held in the right hand. The experimenter (the second author) sat $2.5 \mathrm{~m}$ behind and slightly to the right of the subject, where she controlled the Macintosh II computer. The microswitches were connected to the computer and their state was recorded every ms after the experimenter pressed a key on the computer, at which time she also said 'Go'.

The movement to be executed was alternate tapping of the two switches. Tapping was done with the taped portion of the dowel opposite the end held by the subject. In each trial, the subject was supposed to produce 21 hits ( 20 movements back and forth), alternating between the two switches as quickly as possible. Subjects were free to start the trial on an ad lib basis, there was no reaction time pressure, and there was no requirement to begin the task by hitting a particular switch. After 21 hits were recorded, the computer generated a tone, indicating that tapping could stop. Subjects were told that they would not be penalized if they made more than 21 hits, and they were told not to bother counting the taps. Three successful trials had to be completed in each condition, where a trial was considered successful if 21 hits were completed. After the three successful trials were done, the apparatus was reoriented for the next trial set. Subjects were invited to take short breaks between trials. Although subjects were told to tap as quickly as possible, they were not given immediate feedback about their performance because we did not have a computer program to recognize missed switch closures on-line (see below), which meant that we could not be sure until the data were later analyzed how quickly subjects actually performed correct movements. Subjects were 
told to keep the upper arm against the side of the torso at all times during the oscillation task.

\subsubsection{Design}

In each of the three conditions, the subject completed three practice trials followed by three experimental trials. Altogether, there were 18 experimental trials for each subject ( 2 series $\times 3$ conditions $\times 3$ trials). Once a subject completed all conditions once, he or she did so again in the same order. Thus, the three conditions were tested in two blocks. Each of the six orders of the three conditions was assigned to two subjects, based solely on their order of arrival to the laboratory.

\subsubsection{Data analysis}

The 21 hits on each trial yielded a total of 20 movement times, each of which was defined as the time from the first registration of one switch closure until the first registration of the next switch closure. The data were checked for missed switch closures or switch bounces. Missed switch closures were defined by two successively recorded closures of the same switch with an inter-closure delay approximating the average delay between two closures of the same switch separated by a closure of the other switch. Times corresponding to missed switch closures were excluded from further analysis; there was never more than one such time per trial. Bounces were defined by successively recorded closures of the same switch with an abnormally short inter-closure delay. When a switch bounce was identified, the time between the first closure of that switch and the first closure of the other switch defined the movement time, and this time was used for later analysis.

\subsection{Results}

Movement times, averaged over subjects and over the three experimental trials, are shown for each of the three experimental conditions in Fig. 5. Table 1 shows the same

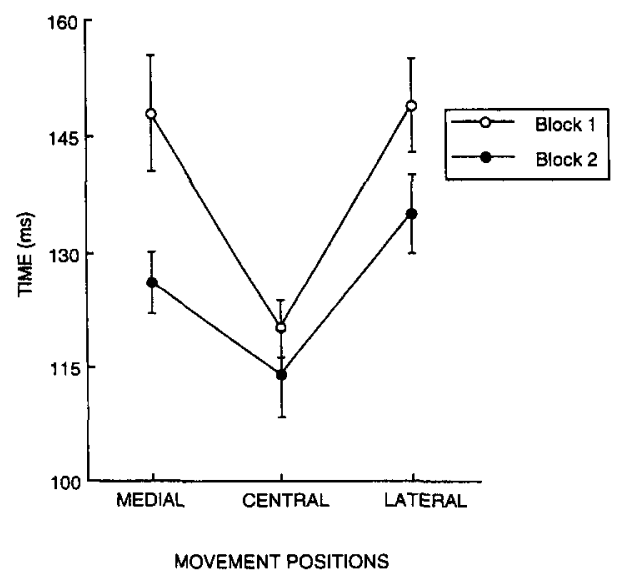

Fig. 5. Mean times for movements in the three ranges tested in Blocks 1 and 2 of Experiment 2. Bars reflect \pm 1 standard error of estimate of the mean, based on subject variability. 
Table 1

Mean times (ms) for supinating (S) and pronating (P) movements in the medial, central, and lateral portions of the movement range. Data from Experiment 2

\begin{tabular}{|c|c|c|c|c|c|c|}
\hline & \multicolumn{6}{|c|}{ Movement positions } \\
\hline & \multicolumn{2}{|c|}{ Medial } & \multicolumn{2}{|c|}{ Central } & \multicolumn{2}{|c|}{ Lateral } \\
\hline & $\mathrm{S}$ & P & $S$ & $\mathbf{P}$ & $S$ & $\mathrm{P}$ \\
\hline \multicolumn{7}{|l|}{ Block } \\
\hline 1 & 150 & 147 & 125 & 114 & 160 & 138 \\
\hline 2 & 126 & 127 & 115 & 114 & 138 & 132 \\
\hline Mean & 138 & 137 & 120 & 114 & 149 & 135 \\
\hline
\end{tabular}

data, but includes movement times for the two directions of movement (supinating and pronating) within each part of the range. Both in Fig. 5 and Table 1, and in all subsequent analyses, data from subjects who used the left hand are combined with data from subjects who used the right hand so that anatomically equivalent conditions go together; thus, the terms 'medial', 'central', and 'lateral' refer to pronated $\left(15^{\circ}\right.$ to $\left.45^{\circ}\right)$, midrange $\left(75^{\circ}\right.$ to $\left.105^{\circ}\right)$ and supinated $\left(135^{\circ}\right.$ to $\left.165^{\circ}\right)$ positions, respectively. Likewise, pronating movements and supinating movements have the same anatomical meaning for all subjects.

An analysis of variance was performed to evaluate the effect of Block ( 1 or 2 ), Position of Motion (medial, central, or lateral), and Direction of Motion (supinating or pronating). The main effect of Block was statistically significant, $F(1,11)=5.20$, $p<0.05$. Movement times were $14 \mathrm{~ms}$ shorter in Block 2 than in Block 1 . The effect of Position of Motion was also statistically significant $F(2,22)=5.21, p<0.02$. As predicted, movement times were shorter in the middle of the range of motion than in either extreme. The mean movement time in the central positions was $117 \mathrm{~ms}$, whereas the mean movement time in the medial and lateral positions were 137 and $142 \mathrm{~ms}$, respectively. The two-way interaction between Block and Position of Motion was statistically significant, $F(2,22)=4.11, p<0.04$. Whereas mean movement times were $29 \mathrm{~ms}$ longer in the medial and lateral positions than in the central position in Block 1, mean movement times were only $17 \mathrm{~ms}$ longer in the medial and lateral positions than in the central position in Block 2. Another way of describing the interaction is to say that, between Block 1 and Block 2, mean movement time decreased by $22 \mathrm{~ms}$ in the medial position, by $14 \mathrm{~ms}$ in the lateral position, and by only $6 \mathrm{~ms}$ in the central position.

The analysis of variance also revealed an interaction between Block and Direction of Motion, $F(2,22)=6.86, p<0.03$. Within Block 1, there was a tendency for pronating movements to be completed more quickly than supinating movements. The direction effect within the lateral part of the range of motion was significant $(p<0.05$, Newman-Keuls test), but was not significant within the central or medial part of the range ( $p>0.05$, Newman-Keuls test). In Block 2 there were no significant direction differences. A separate analysis of variance, limited to Block 2, confirmed that, within the second block, the effect of direction was not significant, $F<1$, nor was the direction $\times$ range interaction, $F<1$, although the effect of Position of Motion was 
significant, $F(2,22)=4.46, p<0.03$. By contrast, an analysis of variance limited to Block 1 revealed a significant effect of Direction, $F(1,11)=6.22, p<0.03$, as well as a significant effect of Position of Motion, $F(2,22)=5.39, p<0.02$, and a nonsignificant interaction between Direction and Position of Motion, $F(2,22)=1.42, p>0.25$.

\subsection{Discussion}

The results of Experiment 1 support the middle-is-faster hypothesis. As predicted, higher frequencies of oscillation were obtained in the middle of the range of motion (the central positions) than in the extreme parts of the range (the medial and lateral positions). This was true both in Block 1 , when subjects were relatively unpracticed, and in Block 2, when they were more practiced. Overall, the movement times were shorter in Block 2 than in Block 1, consistent with the view that subjects benefited from practicing in the second block. The fact that greater speed was observed in the midrange positions than in the extreme positions, both in Block 2 and in Block 1, supports the view that the middle-is-faster effect is a robust property of motor performance.

\section{Experiment 3}

The third experiment had two aims. One was to replicate the results of Experiment 2. The other was to evaluate the generality of the results by testing the middle-is-faster hypothesis both in the nonpreferred and preferred hand. (Recall that only the preferred hand was tested in Experiment 2.) Evaluating the middle-is-faster hypothesis in both hands permitted a test of the idea that biomechanical factors were responsible for the superiority of midrange movements. Insofar as the muscles and tendons of the two arms are functionally identical in typical subjects, the benefit of moving in the middle of the range of motion should be the same regardless of whether the task is carried out with the preferred or nonpreferred hand.

\subsection{Method}

The method was essentially the same as in Experiment 2. Six right-handed students from the University of Massachusetts participated (mean age 23.8 years, range 22 to 29). None of the subjects had served in Experiment 2. Like the subjects of Experiment 2, all were naive with respect to the aims of the study, had normal or corrected-to-normal vision, and had no known neurological or motor disorders.

There were six conditions, created by crossing the three movement positions (medial, central, and lateral) with the two hands. The six conditions were tested in a different random order for each subject. Each condition was tested in six practice trials, followed by three experimental trials. In contrast to Experiment 2, where each condition was tested twice, each condition was tested only once. This aspect of the design, coupled with the number of trials administered in each condition, kept the total number of trials the same as in Experiment 2. As in the first experiment, 21 switch closures had to be registered in a trial to keep the trial from being rerun. The analyses of data proceeded in the same way as in the second experiment. 


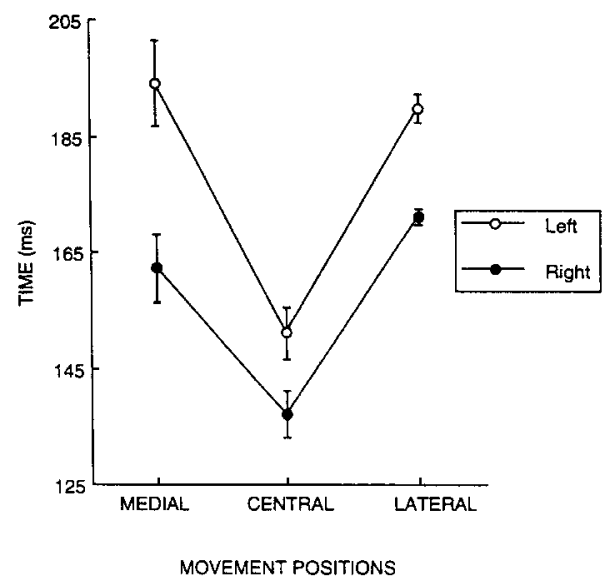

Fig. 6. Mean times for movements performed with the left and right hands in the three ranges tested in Experiment 3. Bars reflect \pm 1 standard error of estimate of the mean, based on subject variability.

\subsection{Results}

Fig. 6 shows the results averaged over subjects and movement directions. Table 2 includes the direction effects. As before, the data for the two hands are collapsed according to anatomical criteria (i.e., 'medial', 'central', and 'lateral' are defined with respect to the body midline, as are 'supinating' and 'pronating').

The movement times were subjected to an analysis of variance that tested the effect of Hand (left or right), Position of Motion (medial, central, or lateral), and Direction of Motion (supinating or pronating). The interaction between Hand and Position of Motion was not statistically significant, $F(2,10)=1.57, p>0.25$. However, both the main effect of Hand, $F(1,5)=23.96, p<0.01$, and the main effect of Position of Motion were statistically significant, $F(2,10)=19.99, p<0.001$. Movement times of the left hand were $21 \mathrm{~ms}$ longer on average than movement times of the right. The mean movement time in the central positions was $145 \mathrm{~ms}$, whereas the mean movement times in the medial and lateral positions were 179 and $181 \mathrm{~ms}$, respectively. As tested with the

Table 2

Mean times (ms) for supinating (S) and pronating (P) movements in the medial, central, and lateral portions of the movement range with the left or right hand. Data from Experiment 3

\begin{tabular}{|c|c|c|c|c|c|c|}
\hline & \multicolumn{6}{|c|}{ Movement positions } \\
\hline & \multicolumn{2}{|c|}{ Medial } & \multicolumn{2}{|c|}{ Central } & \multicolumn{2}{|c|}{ Lateral } \\
\hline & $\mathbf{S}$ & $\mathrm{P}$ & $S$ & $\mathrm{P}$ & $\mathrm{S}$ & $\mathrm{P}$ \\
\hline \multicolumn{7}{|l|}{ Hand } \\
\hline Left & 208 & 180 & 156 & 146 & 202 & 178 \\
\hline Right & 167 & 158 & 142 & 133 & 179 & 163 \\
\hline Mean & 188 & 169 & 149 & 140 & 191 & 171 \\
\hline
\end{tabular}


Tukey HSD procedure, the mean movement time for the central positions was significantly different from the mean movement times for both of the extreme positions ( $p<0.05$ in both cases), although the mean movement times for the two extreme positions did not differ significantly.

Concerning the main effects and interactions involving Direction of Motion, there was a marginally significant two-way interaction of Direction of Motion and Position of Motion, $F(2,10)=3.22, p<0.09$. As seen in Table 2, there was a larger effect of Direction in the medial and lateral positions as compared to the central position. Overall, pronating movements were faster than supinating movements, $F(1,5)=13.02, p<0.02$. Pronating movements had a mean time of $160 \mathrm{~ms}$ whereas supinating movements had a mean time of $176 \mathrm{~ms}$. The interaction between Direction and Hand was not statistically significant, $F(1,5)=1.70, p>0.20$, nor was the three-way interaction of Direction, Hand, and Position of Motion, $p>0.65$. Simple-effects tests showed that the effect of Direction was significant for the lateral positions (when the forearm was most supinated) and for the medial positions (when the forearm was most pronated); the corresponding statistics were $F(1,5)=27.61, p<0.01$, and $F(1,5)=2.20, p<0.02$, respectively. However, the effect of Direction was not significant for the central positions; the corresponding statistic had a $p$-value greater than 0.15 .

\subsection{Discussion}

The third experiment provided further support for the middle-is-faster hypothesis. As in Experiment 2, subjects oscillated the preferred hand at higher frequencies in central positions than in extreme (most supinated or pronated) positions. In addition, the benefit of performing in central positions with the nonpreferred hand was found to be statistically equivalent to the benefit of performing in central positions with the preferred hand. The latter result corroborates the view that the middle-is-faster effect is traceable to physical properties of the arm rather than to differences related to the control of the two arms. We will return to this point in the General Discussion section.

There were two other noteworthy results of the third experiment. First, the nonpreferred (left) arm was slower than the preferred (right) arm. Previous research has shown that greater movement speed is typically obtained with the preferred hand than with the nonpreferred hand (Annett et al., 1958; Annett and Sheridan, 1973; Annett et al., 1979; Flowers, 1975; Kimura and Davidson, 1975), so this result was expected.

Second, there was a tendency for the pronating movements to be quicker than the supinating movements in the medial and lateral positions, though not in the central positions. This outcome is similar to one obtained in Experiment 2. There, pronating movements were quicker than supinating movements in the lateral part of the range of motion, at least in Block 1. Both experiments therefore indicate that pronating movements are quicker than supinating movements, and the difference between pronating times and supinating times tends to be greater in the lateral positions than in the medial or central positions. The basis for the latter conclusion is that in Experiment 2 the supinating-pronating difference was only significant in the lateral positions (albeit in Block 1 only), and in Experiment 3 the supinating-pronating difference was most statistically robust in the lateral positions. The fact that the supinating-pronating 
difference was only apparent in Block 1 of the second experiment suggests that, with practice, subjects can compensate for the difference. Whether, with practice, subjects can compensate for the difference with the nonpreferred hand as well as with the preferred hand is a topic that can be addressed in the future.

\section{General discussion}

We entered this investigation trying to pinpoint the source of the end-state comfort effect. Earlier studies indicated that subjects were willing to tolerate initial discomfort for the sake of final comfort, both in bar-transport tasks (Rosenbaum et al., 1990, 1992; Rosenbaum and Jorgensen, 1992) and in handle rotation tasks (Rosenbaum et al., 1992). We were led to believe that the source of the end-state comfort effect was subjects' expectation that back-and-forth movements would be quicker, and hence more efficient for positioning, in the middle of the range of motion than at either extreme. This led us to predict, in Experiment 1, that the magnitude of the end-state comfort effort would be diminished if we reduced the precision requirements of the handle rotation task. We based this prediction, assuming that when there are minimal precision requirements for final positioning, the need for rapid back-and-forth movements in the homing in phase would be minimized and that subjects would therefore not be concerned with choosing a grip that ensured rapid homing-in movements. This prediction was largely confirmed: When subjects merely had to turn the handle in the direction of a target, so a bolt would drop into a hole at that target, only half the subjects showed the end-state comfort effect. In all previous experiments, where precise end positioning was required, all subjects had shown the effect.

Having shown in Experiment 1 that subjects' grip choices depended on precision requirements, we sought in Experiments 2 and 3 to show that movements are in fact quicker in the middle of the range of motion than at the extremes. There was suggestive evidence for this claim in Experiment 1; handle rotations that ended with the thumb at the most extreme position (position 4) took longer than any other handle rotations. In Experiments 2 and 3, we showed that subjects could in fact oscillate the forearm more quickly in the middle of the range of motion than near the extremes of the range of motion. We called this the middle-is-faster effect.

These results lead us to believe that the source of the end-state-comfort effect is in fact implicit knowledge of the middle-is-faster effect. In these final sections, we ask why, from a biomechanical perspective, the middle-is-faster effect exists. Then we consider the implications of our study for the relation between cognition and biomechanics and for human factors.

\subsection{Why is the middle faster?}

Why can movements be made more quickly in the middle of the range of motion than in the extremes? To answer this question, we need to examine the mechanical basis of the motion itself. Movement times will be dictated by the angular version of Newton's law of acceleration, which states that angular acceleration, $\alpha$, is directly proportional to 
the summation of all torques, $\Sigma T$, acting on the system. This proportionality may be expressed as an equality by considering the system moment of inertia, $I$ :

$$
\Sigma T=I \alpha \text {. }
$$

According to this equation, high torque production and high angular accelerations lead to reduced movement times.

In pronating and supinating arm movements, torques arise from three basic sources: muscles, ligaments and bones. Torques arising from ligaments and bones are passive and provide a source of elastic recoil at the extremes of the range of motion. Muscular torques arise from active muscle forces, and are under the control of the nervous system. Several mechanical factors dictate the capability of the muscles to generate torque at any given angular position, such as the force-length relation of muscle (Gordon et al., 1966), the number and relative strength of muscles involved as either protagonists (assisting the motion) or antagonists (resisting the motion), and the muscle moment arms which convert muscle force into torque. If these factors are considered in total, the torque capacity of each muscle group may be assessed. In pronating movements, the main muscles involved are pronator teres and pronator quadratus, while in supinating movements the main muscles are supinator and biceps brachii. Winters and Kleweno (1993) have demonstrated that the isometric torque patterns for supination and pronation are mirror images of one another, with the supinators being strongest in positions of extreme pronation (medial positions) and the pronators being strongest in positions of extreme supination (lateral positions) (see Fig. 7).

To understand how these mechanical issues impinge on movement times, we must examine the alternating motions of supination and pronation of the forearm. Consider the motion starting from the midpoint of a range of possible positions in either of the latter two experiments. Suppose the initial motion is supination. The forearm is accelerated by the supinating muscles to initiate the motion, but at the end of this cycle

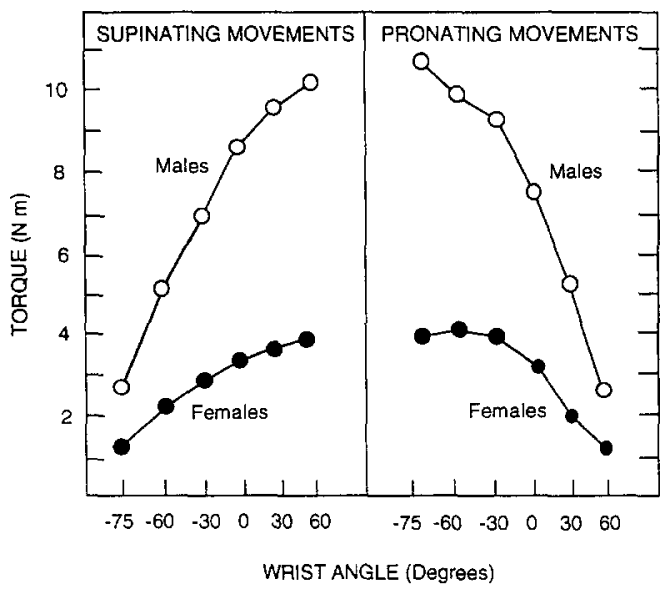

Fig. 7. Torque-angle curves for supination and pronation. Wrist angles are positive when the wrist is pronated. Adapted from J.M. Winters and D.G. Kleweno, 1993. Effect of initial upper-limb alignment on muscle contributions to isometric strength curves. Journal of Biomechanics 26, 143-153. 
the forearm must be halted and the motion reversed. The reversal is accomplished by using the pronating muscles to decelerate the rotation of the forearm until the end of the supination phase is reached, at which time the pronating muscles must initiate acceleration of the forearm in the opposite direction. This acceleration continues across the permitted movement range until the supinating muscles act to decelerate the forearm to stop the motion in the pronation direction, at which time the movement must be reversed and the supinating muscles must accelerate the forearm toward the opposite target. This alternation of supination and pronation must be repeated until the requisite number of movement cycles has been completed. Within each cycle, the time for movement in either direction will be affected by both muscle groups, one for acceleration and the other for deceleration.

We come now to our explanation of the middle-is-fastest effect. Because subjects in Experiments 2 and 3 tried to move as quickly as possible between the two test positions, it would have been adaptive for them to use the highest torques possible for both directions of movement. High torques would have permitted high acceleration, which in turn would have afforded quick movement. It would have also been adaptive for our subjects to use approximately equal torques in the two directions, to prevent one movement from overwhelming the other - that is, to prevent a forceful movement in one direction from being followed by a much less forceful movement in the other direction; the latter movement would have taken a long time to begin, given the inertia associated with the earlier, high-force movement. The best strategy, then, would have been to alternate between opposing torques satisfying two requirements: (1) that the torques were both as high as possible; and (2) that the torques were approximately equal. Given the torque-angle relation shown in Fig. 7, it would have been possible to satisfy these requirements with high torques in the middle of the range of motion, but only with low torques at either of the extremes. At the extreme positions, a high torque would have been possible in one direction, but only a low torque would have been possible in the opposite direction, and so, to satisfy the second requirement of our strategy, the necessary compromise would have been to use the same low torque level in both directions. The upshot would have been slower movements in the extreme positions than in the middle positions. ${ }^{4}$

It is possible to do some rough calculations to get a sense of the torques produced here. During the majority of the motion, the only acting torque is inertial, associated with angular accelerations of the forearm and stick. From the movement times and dimensions of the apparatus, one can estimate the peak angular accelerations during the oscillation movement. For example, using the value of $117 \mathrm{~ms}$, which is the mean

\footnotetext{
${ }^{4}$ Because this model assumes the application of a braking force as well as a driving force, it helps satisfy the concern that the middle-is-faster effect might only hold when subjects perform oscillatory movements which result in collision of a hand-held manipulandum with an obstacle. A possible concern is that the middle-is-faster effect would not be obtained if subjects performed a 'free oscillation' task that did not entail such collisions, and this would be damaging to the view that subjects in the original bar-transport and handle rotation tasks sought to capitalize on the middle-is-faster effect. Our model predicts that quicker oscillations should be observed at midrange even without collisions because the speed benefit of midrange oscillations derives from inherent properties of the muscles balancing the driving and braking forces they can achieve.
} 
movement time for the midrange movements in Experiment 2, the estimated peak acceleration is $76.5 \mathrm{rad} / \mathrm{s}^{2}$. Using an estimated moment of inertia, $I$, of the forearm and stick of $0.01 \mathrm{~kg} \cdot \mathrm{m}^{2}$ (Chapman et al., 1985) the peak inertial torque is $0.765 \mathrm{Nm}$. By contrast, the estimated inertial torque for movements in the medial positions of the range (assuming a mean movement time of $138 \mathrm{~ms}$ and estimated peak acceleration of 55.0 $\mathrm{rad} / \mathrm{s}^{2}$ ) is $0.55 \mathrm{Nm}$, and the estimated torque for movements in the lateral positions of the range (assuming a mean movement time of $142 \mathrm{~ms}$ and peak acceleration of 52.0 $\mathrm{rad} / \mathrm{s}^{2}$ ) is $0.52 \mathrm{Nm}$. These values are dramatically smaller than the isometric values reported by Winters and Kleweno (1993) [see Fig. 7], which is expected because these inertial torques are produced with high contractile velocities rather than isometric contractions.

We believe the capacity for higher torques at mid-range positions provides the simplest explanation for the middle-is-faster effect, although we acknowledge that there may have also been some role for muscle elasticity (even though we doubt that elasticity is the main source of the end-state-comfort effect). The movement patterns in the present experiments had muscle kinematics of alternating concentric/eccentric periods; such alternation is known as the stretch/shorten cycle (Shorten, 1987). This pattern allows the storage of elastic energy in the eccentric (stretching) phase. The energy may then be re-used in the following concentric (shortening) phase to help accelerate the arm (Chapman et al., 1985). The amount of energy stored and subsequently released is directly related to the force level of the muscle. Because the force levels are highest in the mid-range positions, the elastic contribution would have been greater in the central positions than at either extreme. At this time, we cannot say which factor contributed more to the middle-is-faster effect - the capacity for greater bidirectional torques at midrange, or the capacity to store and reuse more elastic energy. Because the two factors are complementary, and because resolution of this issue is not crucial for the main aims of this study, we leave this topic for future research.

\subsubsection{Times for pronating versus supinating movements}

One other open question is why pronating movements tended to be quicker than supinating movements. Recall that movements in the pronating direction had statistically shorter times than movements in the supinating direction, both in Experiment 3 and in Block 1 of Experiment 2. Considering the mean supinating and pronating times shown in Tables 1 and 2, one sees that mean times for pronating movements were shorter than mean times for supinating movements in 11 out of 12 experimental conditions. There was also a hint of such a difference in the handle rotation times of Experiment 1. Handle rotation times were longer for final thumb position 4 than for any other final thumb position; final thumb position 4 was reached by supinating the forearm. By contrast, handle rotation times for final thumb position 5 were not discernibly longer than for other final thumb position; final thumb position 5 was reached by pronating the forearm. Hence, all three experiments yielded evidence, some more direct than others, that pronating movements are faster than supinating movements.

The difference between the times for the two types of movement is an interesting incidental finding of this study. According to our analysis of the muscle activity needed for alternating pronation/supination, pronators and supinators are involved in move- 
ments in both directions, due to the mechanics of accelerating and then decelerating the forearm. Therefore, the shorter movement times for pronation could have been come about because of differences in either the acceleration phase (pronating muscles acting concentrically) or the deceleration phase (supinating muscles acting eccentrically). Either or both of these phases could have been shorter in pronating movements than in supinating movements for any of a number of possible reasons, including differences in neuromuscular excitation times, differences in the proportion of fast twitch motor fibers, differences in torque potentials at different points in the range of motion, or differences in concentric/eccentric force-velocity characteristics. It is beyond the scope of this study to determine which factor(s) is most important, however.

\subsubsection{The role of gravity}

There is another question about the dynamics of the movements required in these studies which warrants discussion. This is whether gravity may have introduced the need to exert a finer degree of control in the medial and lateral conditions than in the central condition in Experiments 2 and 3. In the medial and lateral conditions, the stick's motion was aided by gravity in one direction and was counteracted by gravity in the other direction, but in the central condition, the stick's motion was aided or counteracted by gravity to an equal extent in both directions. Conceivably, the difference in the force requirements for the two directions of motion in the medial and lateral conditions caused subjects to slow down.

There are several reasons why we doubt that this explains our results. First, as seen above, there was a considerable agreement between the results of Experiment 1, where the wheel turned in the horizontal plane, and the results of Experiments 2 and 3, where the stick was rotated in the gravitational plane. Moreover, the earlier study by Rosenbaum et al. (1993) showed that handle rotation performance is unaffected by the orientation of the plane in which a manipulandum must be rotated. Second, if subjects' performance was primarily related to gravity, one would expect downward rotations to have roughly constant durations, regardless of whether they occurred in the medial or lateral positions; similarly, one would expect upward rotations to have roughly constant durations, regardless of whether they occurred in the medial or lateral positions. In fact, the two kinds of downward movements had quite different times, and so did the two kinds of upward movements. Pronations in the medial position (a downward movement) took much less time than supinations in the lateral position (another downward movement): the times were 137 versus $149 \mathrm{~ms}$ in Experiment 2, and 169 versus $191 \mathrm{~ms}$ in Experiment 3. Similarly, the two kinds of upward movements had quite different times, at least in Block 1 of Experiment 2 and in Experiment 3. In Block 1 of Experiment 2 supinations in the medial position took longer than pronations in the lateral position (150 versus $138 \mathrm{~ms}$ ), and the same was true in Experiment 3 (188 ms versus $171 \mathrm{~ms}$ ).

The third argument against the gravity explanation is that other studies of manual performance suggest that the effects of gravity on the kinematics of arm movements are

\footnotetext{
${ }^{5}$ We thank Gerard van Galen for suggesting this interesting hypothesis.
} 
surprisingly small. Uno et al. (1989), in their classic study introducing the minimum torque-change optimization model, reported that the kinematics of upward and downward arm movements in the sagittal plane were nearly indistinguishable (one movement could be viewed as the mirror image of the other). They also found that the kinematics of the arm in these vertical movements was hardly affected by different payloads. Uno et al. concluded that gravitational forces had negligible effects on sagittal plane movements of the arm. The latter results have been replicated in informal studies (Zatsiorsky, 1995).

Finally, calculation of the gravitational torque in the middle of the medial or lateral target range yields a value of $0.096 \mathrm{Nm}$, which is only about $12 \%$ of the highest estimated inertial torque. It seems unlikely that this small additional torque due to gravity could have been enough to completely explain our data.

In sum, although none of these results proves that gravity played no role in the oscillatory movements of the subjects studied here, they suggest that little weight should to be given to gravity as a means of explaining our results.

\subsection{Biomechanics and cognition}

It is interesting to consider the implications of our results for the relation between biomechanics and cognition. Although both disciplines pertain to motor control, they have rarely been considered together. Our research suggests that they should be. Cognition has usually been concerned with decision making, usually in domains far removed from motor performance. Biomechanics, on the other hand, has usually been concerned with properties of musculoskeletal motion and stability, but with little regard for the way patterns of performance are chosen. How one chooses to act must take into account what one can do, however. At some level, the system responsible for selecting movements must 'know about' the body's biomechanical properties. The finding that the end-state comfort effect may be rooted in biomechanics supports this view. The planning of movements - usually considered a high-level activity in traditional accounts of performance - appears to proceed with considerable information about low-level features of neuromuscular performance, or aspects of movement that depend quite directly on low-level features. This point has been made by others, of course, most notably Bernstein (1967), who argued that as actors become more proficient in a task, they exploit biomechanical properties of the body in its interaction with the external environment; for example, they exploit rather than counteract reactive forces among the

limb segments. For supporting evidence, see Schneider et al. (1989). Our conclusions reinforce this general perspective.

No less important than this message is a message about the route we followed to get to it. The end-state-comfort effect was discovered in a search for regularities in macroscopic behavioral choices. The observation that people usually grab a stick in an uncomfortable initial posture to end up in a more comfortable final posture was surprising. The phenomenon reflected future planning, and was especially interesting because it implied that the perception of affordances (how best to grab a stick) does not just depend on the stimulus (the image of the stick) or on the actor's current physical state (e.g., the current state of the muscles and joints), but rather on information embodied in the actor's intentional state regarding what he or she wants to achieve (see 
Stelmach et al., 1994, for a similar conclusion). Nevertheless, the question that remained was what information is used by the actor to make the grip choice. In our attempt to track down the relevant parameters, we were led to the discovery of a new, apparently low-level, phenomenon of motor performance - the middle-is-faster effect. It is appealing that this finding about relatively low-level aspects of behavior followed from observations of macroscopic behavioral choices. Coming full circle in this way supports the view that the system responsible for planning macroscopic features of behavior has access to information about low-level features of the body's capabilities.

\subsection{Implications for human factors}

As with any empirical effect, it is important to consider the practical implications of the effects that have been uncovered. Our results suggest that a dial or knob which demands quicker rotation in one direction than another should be oriented so the quicker rotation can be achieved with pronation rather than supination, all else being equal. Similarly, a dial or knob which demands most rapid and/or most forceful oscillation in a certain part of the rotation range should be designed so that part of the range can be covered with the forearm at or near the center of the pronation-supination dimension.

Even if these recommendations need to be made explicit for human-factors engineers, it is our impression that people have implicit knowledge of these facts and act on this implicit knowledge everyday. Informal observations indicate that when people turn dials on locks or on radios they try to complete the tasks with the forearm at or near the middle of the pronation-supination range; indeed, it would be strange to see a person spontaneously carrying out these movements with the arm twisted in an extreme posture. It is not the case that people simply prefer to maintain postures that keep the joints at or near the middle of their ranges of motion (Cruse et al., 1993), for then it would be impossible to move. By itself, the preference to remain at midrange positions does not let one predict when an actor will depart from the middle of the range, as for example, when they deliberately adopt awkward postures prior to transporting an object which then leaves them in a comfortable posture (the end-state comfort effect). Our observations, beginning with the end-state-comfort effect, suggest that people draw on knowledge about the capabilities of their bodies, as embodied in phenomena like the middle-is-faster effect, to make the decisions they do about how to act.

\section{Acknowledgements}

The research was supported by grants BNS-90-08665 and SBR-94-96290 from the National Science Foundation and a Research Scientist Development Award from the National Institute of Mental Health (all to the first author). The authors thank Erin Nano for help with data collection, Michael Bushe for help with computer programming, Charles Gorman and Gaspar DiPasquale for technical support, Loukia Loukopoulos, Horst Krist, Martin Fischer, Lyn Lagasse, and Joe Hamill for helpful discussions, and Geoffrey Bingham, Carol Fowler, Claire Michaels, Gerard van Galen, and two anonymous reviewers for useful comments on earlier versions of this report. 


\section{References}

Alexander, R.M., 1984. Walking and running. American Scientist 72, 348-354.

Annett, J., C.W. Golby and H. Kay, 1958. The measurement of elements in an assembly task: The information output of the human motor system. Quarterly Journal of Experimental Psychology 10, 1-11.

Annett, J., M. Annett, P.T.W. Hudson and A. Turner, 1979. The control of movements in the preferred and non-preferred hands. Quarterly Journal of Experimental Psychology 31, 641-652.

Annett, J. and M.R. Sheridan, 1973. Effects of S-R and R-R compatibility on bimanual movement time. Quarterly Journal of Experimental Psychology 25, 247-252.

Bernstein, N., 1967. The coordination and regulation of movements. London: Pergamon.

Bizzi, E. and F.A. Mussa-Ivaldi, 1989. 'Geometrical and mechanical issues in movement planning and control'. In: M.I. Posner (Ed.), Handbook of cognitive science (pp. 769-792). Cambridge, MA: MIT Press.

Bushe, M.M., J. Vaughan and D.A. Rosenbaum, 1994. Pascal external functions for Strawberry Tree's 'Analog Connection Workbench'. Behavior Research Methods, Instruments, and Computer 26, 461-466.

Chapman, A.E., G.E. Caldwell and W.S. Selbie, 1985. Mechanical output following muscle stretch in forearm supination against inertial loads. Journal of Applied Physiology 59, 78-86.

Crossman, E.R.F.W. and P.J. Goodeve, 1963. Feedback control of hand-movement and Fitts' Law. Quarterly Journal of Experimental Psychology 35A, 251-278.

Cruse, H., M. Brüwer and J. Dean, 1993. Control of three- and four-joint arm movement: Strategies for a manipulator with redundant degrees of freedom. Journal of Motor Behavior 25, 131-139.

Fitts, P.M., 1954. The information capacity of the human motor system in controlling the amplitude of movement. Journal of Experimental Psychology 47, 381-391.

Flowers, K., 1975. Handedness and controlled movements. British Journal of Psychology 66, 39-52.

Gordon, A.M., A.F. Huxley and F.J. Julian, 1966. The variation in isometric tension with sarcomere length in vertebrate muscle fibres. Journal of Physiology 184, 1780-1792.

Holst, E. von, 1939. Die relative Koordination als Phänomen und als Methode zentralnervöser Funktionsanalyse. Erg. Physiol. 42, 228-306. English translation in Holst, E. von, 1973. 'Relative coordination as a phenomenon and as a method of analysis of central nervous functions'. In: The behavioural physiology of animal and man: The collected papers of Erich von Holst (Vol. 1) ([R. Martin, Translator). London: Methuen.

Kimura, D. and W. Davidson, 1975. Right arm superiority for tapping with distant and proximal joints. Journal of Human Movement Studies 1, 199-202.

Kugler, P.N., J.A.S. Kelso and M.T. Turvey, 1980. 'On the concept of coordinative structures as dissipative structures: I. Theoretical lines of convergence'. In: G.E. Stelmach and J. Requin (Eds.), Tutorials in motor behaviour (pp. 3-47). Amsterdam: North-Holland.

McMahon, T.A., 1984. Muscles, reflexes and locomotion. Princeton, NJ: Princeton University Press.

Meyer, D.E., R.A. Abrams, S. Kornblum, C.E. Wright and J.E.K. Smith, 1988. Optimality in human motor performance: Ideal control of rapid aimed movements. Psychological Review 95, 340-370.

Rosenbaum, D.A. and M.J. Jorgensen, 1992. Planning macroscopic aspects of manual control. Human Movement Science 11, 61-69. (Also appeared in A. Thomassen, D.A. Rosenbaum and P.C.W. van Wieringen (Eds.), 1992. Sequencing and timing of movement. Amsterdam: North-Holland.)

Rosenbaum, D.A., J. Vaughan, H.J. Barnes, F. Marchak and J. Slotta, 1990. 'Constraints on action selection: Overhand versus underhand grips'. In: M. Jeannerod (Ed.), Attention and performance XIII (pp. 321-342). Hillsdale, NJ: Lawrence Erlbaum Associates.

Rosenbaum, D.A., J. Vaughan, H.J. Barnes and M.J. Jorgensen, 1992. Time course of movement planning: Selection of hand grips for object manipulation. Journal of Experimental Psychology: Learning, Memory, and Cognition 18, 1058-1073.

Rosenbaum, D.A., J. Vaughan, M.J. Jorgensen, H.J. Barnes and E. Stewart, 1993. 'Plans for object manipulation'. In: D.E. Meyer and S. Kornblum (Eds.), Attention and performance XIV - A silver jubilee: Synergies in experimental psychology, artificial intelligence and cognitive neuroscience (pp. 803-820). Cambridge, MA: MIT Press/Bradford Books.

Schneider, K., R.F. Zernicke, R.A. Schmidt and T.J. Hart, 1989. Changes in limb dynamics during the practice of rapid arm movements. Journal of Biomechanics 22, 805-817.

Shorten, M.R., 1987. Muscle elasticity and human performance. Medicine and Sport Science 25, 1-18. 
Stelmach, G.E., U. Castiello and M. Jeannerod, 1994. Orienting the finger opposition space during prehension movements. Journal of Motor Behavior 26, 178-186.

Thelen, E., J.A.S. Kelso and A. Fogel, 1987. Self-organizing systems and infant motor development. Developmental Review 7, 39-65.

Uno, Y., M. Kawato and R. Suzuki, 1989. Formation and control of optimal trajectory in human multijoint arm movement: Minimum torque-change model. Biological Cybernetics 61, 89-101.

Winters, J.M. and D.G. Kleweno, 1993. Effect of initial upper-limb alignment on muscle contributions to isometric strength curves. Journal of Biomechanics 26, 143-153.

Zatsiorsky, V., 1995, March. Personal communication. 